\title{
THE STRUCTURE OF A SPECIAL CLASS OF RINGS ${ }^{1}$
}

WALLACE S. MARTINDALE, III

A ring $R$ such that for every $x \in R$ there exists an element $c(x) \in R$ (depending on $x$ ) such that $x^{2} c(x)-x$ is in the center will be called a $\xi$-ring [9]. ${ }^{2}$ It is our purpose in the present paper to study the following conjecture of I. N. Herstein: Every $\xi$-ring $R$ is a subdirect sum of rings $R_{\alpha}$ where either (1) $R_{\alpha}$ is a division ring or (2) there exists an ideal $P_{\alpha}$ in the center of $R_{\alpha}$ such that $P_{\alpha}=R_{\alpha}$ or $R_{\alpha} / P_{\alpha}$ is a field. The validity of this conjecture will be shown in the presence of certain additional restrictions on the ring $R$. In general we shall prove a similar theorem in which the possibility (2) is weakened so as to read, "Every commutator of $R_{\alpha}$ is in the center."

1. The general solution. Our $\xi$-ring will be denoted by $R$, the center by $Z$, the (Jacobson) radical by $N$, the set of nilpotent elements by $I$, and the commutator ideal by $C$.

THEOREM 1. If $R$ is a $\xi$-ring, then $x c(x)=c(x) x$ for all $x \in R$.

Proof. ${ }^{3}$ For the sake of convenience we shall write $c$ for $c(x)$. If $a \in R$ satisfies $a^{2}=0$, then since $a^{2} c(a)-a \in Z$ we have $a \in Z$. Let $x \in R$. Since $x^{2} c-x \in Z,\left(x^{2} c-x\right) x=x\left(x^{2} c-x\right)$ and so we obtain (1) $x^{2}(x c-c x)=0$. Using (1) $[x(x c-c x) x]^{2}=0$ and so (2) $x(x c-c x) x \in Z$. Commuting this with $x$ and using (1) we arrive at (3) $x(x c-c x) x^{2}=0$. Since $x^{2} c-x \in Z$ we can easily verify that (4) $x c-c x=[x(x c-c x)+(x c-c x) x] c$. We left multiply (4) by $x$; using (1) this simplifies to (5) $x(x c-c x)=x(x c-c x) x c=c x(x c-c x) x$ by (2). We right multiply (5) by $x$ and so get (6) $x(x c-c x) x=c x(x c-c x) x^{2}=0$ by (3). Reapplying the result of (6) to (5), (5) reduces to (7) $x(x c-c x)=0$. This result simplifies (4) to (8) $x c-c x=(x c-c x) x c$. From $(7)$ it follows that $[(x c-c x) x]^{2}=0$ and so $(x c-c x) x \in Z$; com-

Received by the editors January 29, 1958.

1 The material in this paper is a portion of a dissertation submitted to the University of Pennsylvania under the guidance of Dr. I. N. Herstein. The work on the paper was done at Yale University while the author was a research assistant to Dr. Herstein under a National Science Foundation grant (NSF-G2270).

2 Utumi has shown in [9] that any $\xi$-ring modulo the maximal nil ideal is a subdirect sum of division rings and commutative rings. His arguments are along slightly different lines than those used in this paper.

${ }_{3}$ The proof given here is a direct one due to Dr. Herstein. We mention also that the concept of subdirect irreducibility, first exploited by Herstein in [4], is used strongly in the present paper. 
muting this with $x$ and using (7), we have (9) $(x c-c x) x^{2}=0$. Since $(x c-c x) x \in Z(8)$ becomes $(x c-c x)=c(x c-c x) x$, and so (10) $(x c-c x) x$ $=c(x c-c x) x^{2}=0$ from (9). But then (8) reduces to $x c-c x=0$ which is the desired result.

Lemma 1. Let $x \in R$. Then there exist elements $c_{n} \in R, n=1,2, \cdots$, such that $x^{2 n} c_{n}-x \in Z$.

Proof. The lemma is valid for $n=1$ by setting $c_{1}=c(x)$. For $n=2$ we choose a $d \in R$ such that $\left(x^{2} c_{1}\right)^{2} d-x^{2} c_{1} \in Z$. Applying Theorem 1 , we set $c_{2}=c_{1}^{2} d$ and see that $x^{4} c_{2}-x=x^{4} c_{1}^{2} d-x=\left(x^{2} c_{1}\right)^{2} d-x$ $=\left[\left(x^{2} c_{1}\right)^{2} d-x^{2} c_{1}\right]+\left(x^{2} c_{1}-x\right) \in Z$. Continuing in this fashion, we see that an easy induction will establish the lemma.

As an important corollary we have

Lemma 2. $I$ is a two-sided ideal of $R$ contained in the center $Z$.

The following lemma is true in any ring in which every nilpotent element is central.

Lemma 3. Every idempotent of $R$ is in the center.

TheOREM 2. Every primitive $\xi$-ring $R$ is a division ring.

Proof. Since $T$ is primitive there exists a maximal right ideal $J$ such that $[J: R]=(0)$, where $[J: R]=\{x \in R \mid R x \subset J\}$.

Suppose $J \neq(0)$. A contradiction will result provided we produce in $J$ a nonzero central element, for if $0 \neq z \in J \cap Z$, then $R z=z R \subset J$ and thus $[J: R] \neq(0)$. To this end we choose a nonzero element $a \in J$ and, setting $c=c(a)$, note that $z=a^{2} c-a \in J \cap Z$. Without loss of generality we may assume that $z=0$, in which case $(a c)^{2}-a c$ $=(a c a-a) c=\left(a^{2} c-a\right) c=z c=0$, using Theorem 1. By Lemma 3 the idempotent $a c \in J \cap Z$, and it is nonzero because otherwise $a^{2} c-a$ $=-a \in Z$ and so $0 \neq a \in J \cap Z$. We have completely disposed of the possibility that $J \neq(0)$.

When we consider the case that the maximal right ideal $J$ is zero, it is well known that either $R$ is a division ring or $R^{2}=(0)$, and the latter case is ruled out because $N=(0)$.

COROllary 1. Every semi-simple $\xi$-ring $R$ is a subdirect sum of division rings.

COROllary 2. Every subdirectly irreducible semi-simple $\xi$-ring is a division ring.

Since any ring is a subdirect sum of subdirectly irreducible rings, the second corollary to Theorem 2 reduces our study of $\xi$-rings to 
that of subdirectly irreducible $\xi$-rings with nontrivial radical. $R$ will now be assumed to be subdirectly irreducible with minimal two-sided ideal $S \neq(0)$ and $N \neq(0)$.

Lemma 4. $S^{2}=(0)$ and $S \subset Z$.

Proof. ${ }^{4}$ Let $0 \neq s \in S$ and choose a $c \in R$ such that $s^{2} c-s=z \in Z \cap S$. Since $S \subset N$ we know that $z \neq 0$, for otherwise $s$ would be a radical multiple of itself. Either the two-sided ideal $z S=S$ or $z S=(0)$, by the definition of $S . z S=S$ is impossible, because $z$ would be a radical multiple of itself. Thus $z S=(0)$. Now, choosing any $t \in S$, we see of course that $0=z t=t z=t s^{2} c-t s$. In other words, $(t s)(s c)=t s$, forcing $t s=0$, since $t s$ is a radical multiple of itself. As $s$ and $t$ were completely arbitrary elements of $S$ we have shown that $S^{2}=(0)$. In particular $s^{2}=0$ for all $s \in S$, so by Lemma 2 we may conclude that $S \subset Z$.

In any ring it is easy to verify the following

Lemma 5. If $U$ is any ideal of $R$ contained in the center of $R$, then $C U=(0)$, where $C$ is the commutator ideal of $R$.

Lemma 6. If $x y \in S$ for some $x \in C$ and some $y \in R$, then $x y=y x$.

Proof. Lemma 5 implies that $(y x)^{2}=y[(x y) x]=0$ since $x y \in S \subset Z$ and $x \in C$. Thus $y x$ as well as $x y$ is contained in the ideal $I \subset Z$. We now choose a $c \in R$ such that $x^{2} c-x \in Z$ and, using Theorem 1, write $x y-y x=\left(x^{2} c\right) y-y\left(x^{2} c\right)=c[x(x y)]-[(y x) x] c$. The right hand side is zero since $x \in C, x y \in I$, and $y x \in I$. It follows that $x y=y x$.

Lemma 7. Let $a \in C, y, r \in R$, and $s \in S$ such that $s=(a y-y a) r$. Then $s=0$.

Proof. We select an element $c \in R$ such that $a^{2} c-a \in Z$ and write $v=a y-y a=a^{2} c y-y a^{2} c=a^{2}(c y-y c)+a(a y-y a) c+(a y-y a) a c$. This means that $s=v r=a^{2}(c y-y c) r+a v c r+v a c r$. Noting that $S \subset Z$ and $v$, $a c, v a c \in C$, we see by Lemma 5 and Lemma 6 that $0=s(a c)=(v r)(a c)$ $=(r v)(a c)=r(v a c)=(v a c) r$. A similar argument shows that $a v c r=0$. We are thus left with $0 \neq s=a^{2}(c y-y c) r$. Again, using Lemma 5 and Lemma 6, we have $0=s(c y-y c) r=[a(a(c y-y c) r)](c y-y c) r$ $=[(a(c y-y c) r) a](c y-y c) r=[a(c y-y c) r]^{2}$. By Lemma $2 a(c y-y c) r$ $\in I \subset Z$. $s=a[a(c y-y c) r] \in C I=(0)$ by Lemma 5 , and the proof is complete.

THEOREM 3. If $R$ is a subdirectly irreducible $\xi$-ring with nontrivial radical, then every commutator of $R$ is in the center.

- See Herstein [6, p. 107]. 
Proof. Let $a=e f-f e$ be a commutator of $R$, and, if $y \in R$, consider the element $v=a y-y a$. We choose an element $d \in R$ such that $v^{2} d-v \in Z$ and form the two-sided ideal $\left(v^{2} d-v\right) R$. If $\left(v^{2} d-v\right) R \neq(0)$, then $S \subset\left(v^{2} d-v\right) R$, yielding $0 \neq s=\left(v^{2} d-v\right) t=v r$ for suitable $s \in S$ and $t, r \in R$, a contradiction of Lemma 7. If $\left(v^{2} d-v\right) R=(0)$, then in particular $\left(v^{2} d-v\right) d=0$, and, making use of Theorem $1, v d$ is an idempotent. By Lemma $3, v d \in Z$ and so $v d R$ is a two-sided ideal. $v d R \neq(0)$ is again ruled out because of Lemma 7 , and, recalling that $\left(v^{2} d-v\right) R=(0)$, we have $v R=(a y-y a) R=(0)$. Since $a e e(f e)-(f e) e$ is also a commutator, we may replace $a$ by $a e$ in the preceding discussion and obtain $[(a e) y-y(a e)] R=(0)$. In particular $a^{3}=a(e f-f e) a$ $=(a e f-f a e) a+(f a e-a f e) a=[(a e) f-f(a e)] a+[(f a-a f) e] a=0$. Using Lemma 2, we have finally $a \in Z$.

We now consider any arbitrary $\xi$-ring $R$ and remark that it can be written as a subdirect sum of subdirectly irreducible $\xi$-rings $R_{\alpha}$, since homomorphic images of $\xi$-rings are again $\xi$-rings. Applying Theorem 3 and Corollary 2 of Theorem 2 we have finally proved the

MaIN Theorem. Every $\xi$-ring $R$ is a subdirect sum of subdirectly irreducible rings $R_{\alpha}$ where either $R_{\alpha}$ is a division ring or every commutator of $R_{\alpha}$ is in the center of $R_{\alpha}$.

We shall again confine our attention to subdirectly irreducible $\xi$-rings with nontrivial radical. Theorem 3 tells us that every commutator is central, and it is well known that in this situation $C^{2}=(0)$. Therefore we now have $(0) \neq S \subset C \subset I \subset N \cap Z$, provided $R$ is not already commutative.

Let $x, y \in R$. We assert that $x y=y x+a y x$, where $a=a(x, y) \in C$. In fact, if $c, d \in R$ such that $x^{2} c-x \in Z$ and $y^{2} d-y \in Z$, we can write $x y-y x=x^{2}(c y-y c)+2 x c(x y-y x)=x^{2}(y c-c y)(2 y d)+x^{2} y^{2}(d c-c d)$ $+2 x y(y x-x y)(2 y d)+(2 x c) y^{2}(d x-x d)=a y x$, making liberal use of $C \subset Z$. An immediate corollary to this result is that every right (left) ideal of $R$ is two-sided. In case $R$ has an identity element we have shown that $x y=\lambda y x$ for all $x, y \in R$, where $\lambda=\lambda(x, y)=1+a$ is an invertible central element, since $a \in N$.

Let $A(S)$ be the set $\{a \in R \mid a S=(0)\}$. We shall develop briefly some results already studied by Herstein [6, pp. 109-110]. Our first assertion is that $A(S)$ coincides with the union of left and right zero divisors of $R$. Indeed, let $a \neq 0 \in R$ be $a$ (left) zero divisor, i.e., $a b=0$ for some $b \neq 0$. If $T=\{x \in R \mid a x=0\}$, then $(0) \neq S \subset T$ since $T$ is a nonzero right ideal; therefore $a S=(0)$.

We claim next that $N S=(0)$. Indeed, suppose that $N S$, being an ideal contained in $S$, is equal to $S$. Then for some nonzero element 
$s \in S$ we have $N s \neq(0)$ and hence $N s=S$. A contradiction is then reached since $s$ would be a radical multiple of itself. Thus $N \subset A(S)$ and we can extend our information about $R$ to $(0) \neq S \subset C \subset I \subset Z \cap N$ $\subset N \subset A(S)$.

We finally prove that either $R=A(S)$ or else $\bar{R}=R / A(S)$ is a field. Suppose that $R \neq A(S) . \bar{R}$ is commutative since $C \subset A(S)$. There exists an $s \neq 0 \in S$ such that $s R \neq(0)$ (because $R \neq A(S)$ ), and hence $S=s R$. Choosing an $e \in R$ such that $s e=s$, we see that $e x-x \in A(S)$ for all $x \in R$, since $s(e x-x)=(s e) x-s x=0$ and all zero divisors are in $A(S)$. It follows that the element $\bar{e}$ serves as the identity in $\bar{R}$. Now suppose $x \in A(S)$. Then $0 \neq x s=t \in S$, and, from $t R \neq(0)$, we can select a $y \in R$ such that $t y=s$. Thus $(x y) s=(x s) y=t y=s$ and $(x y) s=(x y)(s e)=s e$, or $(x y-e) s=0$. This shows that $x y-e \in A(S)$ and that $\bar{y}$ is an inverse for $\bar{x}$. The assertion that $\bar{R}$ is a field or $A(S)=R$ has now been demonstrated.

We remark finally in this section that all our results for $\xi$-rings up to this point could just as well have been derived for $\xi$-algebras.

2. Special cases. Before beginning our study of certain special $\xi$-rings we state a simple but well known result in ring theory which will be of general use throughout the present section.

LEMma 8. Let $R$ be either a ring or an algebra (over a field $\Phi$ ), and suppose for some $x, y \in R$ that $x y-y x$ is central. Let $p(x)=\sum_{i=0}^{n} \alpha_{i} x^{i}$ be a polynomial in $x$, where the $\alpha_{i}$ are integers (if $R$ is a ring) or the $\alpha_{i} \in \Phi$ (if $R$ is an algebra). Denote by $p^{\prime}(x)=\sum_{i=1}^{n} i \alpha_{i} x^{i-1}$ the formal derivative of $p(x)$. Then $p(x) y-y p(x)=p^{\prime}(x)(x y-y x)$.

We shall next study $\xi$-rings $R$ under the restriction that for all $x \in R c(x)$ is in the subring $(x, Z)$ generated by $x$ and the center $Z$. Since the quaternions satisfy this property, our main hope of obtaining a sharper picture of $R$ lies in considering the case where $R$ is subdirectly irreducible with nontrivial radical.

THEOREM 4. Let $R$ be a $\xi$-ring in which $S \neq 1(0), N \neq(0)$, and $c(x) \in(x, Z)$ for all $x \in R$. Then $A(S) \subset Z$ and either $R$ is commutative or $R / A(S)$ is a field.

Proof. Suppose $x \in A(S)$. By assumption $c=c(x)=\sum_{i=0}^{n} z_{i} x^{i}$ for suitable $z_{i} \in Z$. Let $y \in R$. Then $x y-y x=\left(x^{2} c\right) y-y\left(x^{2} c\right)$

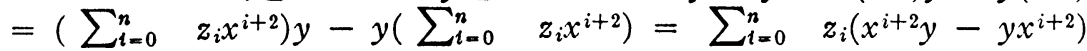
$=\sum_{i=0}^{n}(i+2) z_{i} x^{i+1}(x y-y x)=a(x y-y x)$ where $a \in A(S)$, using the fact that $x y-y x \in Z$ by Theorem 3 and applying Lemma 8 to $x^{i+2} y-y x^{i+2}$ for all $i$. If $(x y-y x) R=(0)$, then $x y-y x=0$ by the equation $x y-y x=a(x y-y x)$. If $(x y-y x) R \neq(0)$ we can find an $r \in R$ 
such that $0 \neq s=(x y-y x) r$, and a contradiction arises if we note that $(x y-y x) r=a(x y-y x) r=a s=0$ since $a \in A(S)$. Hence $x y-y x=0$ for all $x \in A(S)$ and all $y \in R$, that is, $A(S) \subset Z$. Clearly $R$ is commutative if $R=A(S)$, and, if $R \neq A(S)$, we know already from the previous section that $R / A(S)$ is a field.

Theorem 4A. Let $R$ be a $\xi$-ring in which $c(x) \in(x, Z)$ for all $x \in R$. Then $R$ is a subdirect sum of subdirectly irreducible rings $R_{\alpha}$ where either (1) $R_{\alpha}$ is a division ring or (2) there exists an ideal $P_{\alpha}$ of $R_{\alpha}$ contained in the center of $R_{\alpha}$ such that $P_{\alpha}=R_{\alpha}$ or $R_{\alpha} / P_{\alpha}$ is a field.

Proof. We first apply the Main Theorem and note that the condition $c(x)$ carries over to the homomorphic images $R_{\alpha}$. The proof is then immediate if for each $R_{\alpha}$ we set $P_{\alpha}=A(S)$ and apply Theorem 4 .

Theorem 4 generalizes a result of Herstein [7, p. 868, Theorem 11]; in his paper $c(x)$ is a polynomial in $x$ with integer coefficients. Our next specialization occurs in

THEOREM 5. Let $R$ be an algebraic $\xi$-algebra over a field $\Phi$, in which $S \neq(0)$ and $N \neq(0)$. Then $I=N=A(S) \subset Z$, and $R$ is either commutative or $R / A(S)$ is a field.

Proof. If $A(S) \neq I$ there is an $a \in A(S)$ such that $a$ is not nilpotent. Since $R$ is algebraic we can write $\sum_{i=0}^{n} \alpha_{i} a^{i}=0$ for some $n \geqq 1$, where $\alpha_{n}=1$ and $\alpha_{i} \in \Phi$ for $i=0,1, \cdots, n . \alpha_{0}$ must be zero, for otherwise, choosing some $s \neq 0 \in S$, we would have $0=\left(\sum_{i=1}^{n} \alpha_{i} a^{i}\right) s=-\alpha_{0} s$, a contradiction. Therefore we now have $\sum_{i=m}^{n} \alpha_{i} a^{i}=0$, where $m \geqq 1$ and $\alpha_{m} \neq 0$. If $a^{m} R \neq 1(0)$, then $S \subset a^{m} R$, so we can choose an $r \in R$ such that $0 \neq s=a^{m} r \in S$. Then $0=\left(\sum_{i=m}^{n} \alpha_{i} a^{i}\right) r=\alpha_{m} a^{m} r+\sum_{i=1}^{n-m} \alpha_{m+i} a^{i}\left(a^{m} r\right)$ $=\alpha_{m} s+\sum \alpha_{i} a^{i} s=\alpha_{m} s$ since $a \in A(S)$. This is an impossible situation since $\alpha_{m} \neq 0$ and $s \neq 0$. Thus $a^{m} R=(0)$, and in particular $0=a^{m} a=a^{m+1}$ so $a$ is nilpotent, contradicting our original assumption about $a$. Our argument shows that $A(S) \subset I$, and, since we already know that $I \subset A(S) \cap Z$, we can safely assert that $I=N=A(S) \subset Z$. The rest of the theorem follows easily.

Theorem 5 can be sharpened if we impose the condition that $\Phi$ is perfect.

TheORem 6. Let $R$ be an algebraic $\xi$-algebra over a perfect field $\Phi$ in which $S \neq(0)$ and $N \neq(0)$. Then $R$ is commutative.

Proof. Suppose there exists an $x \in R$ such that $x \in Z$. By Theorem $4 x \in A(S)$, and $\bar{R}=R / A(S)$ is an algebraic extension field of $\Phi$. Let $p(t)=\sum_{t=0}^{n} \alpha_{i} t^{i}$ be the minimal polynomial over $\Phi$ satisfied by $\bar{x} \in \bar{R}$. The degree of $p(t)>1$, for otherwise $\alpha_{0}+x \in A(S)$, forcing $x \in Z$. 
Since $\Phi$ is perfect, $p(t)$ is a separable polynomial, which means that its derivative $p^{\prime}(t)=\sum_{i=1}^{n} i \alpha_{i} t^{i-1}$ is not identically zero. Now let $y \in R$ such that $x y-y x \neq 0$. We now use the fact that $p(x) \in Z$ and $x y-y x \in Z$ (Theorem 3), together with an application of Lemma 8, to write the equation $0=p(x) y-y p(x)=\left(\sum \alpha_{i} x^{i}\right) y-y\left(\sum \alpha_{i} x^{i}\right)$ $=\sum \alpha_{i}\left(x^{i} y-y x^{i}\right)=(x y-y x)\left(\sum i \alpha_{i} x^{i-1}\right)=(x y-y x) p^{\prime}(x)$. It follows that $p^{\prime}(x) \in A(S)$ because $x y-y x \neq 0$. In other words, $p^{\prime}(\bar{x})=0$ in $\bar{R}$, contrary to the minimality of $p(t)$. Therefore we must assume that $R=Z$, and the proof is complete.

Both forms of the Main Theorem corresponding to Theorem 5 and to Theorem 6 can now be sharpened in a natural way.

Theorem 5A. If $R$ is an algebraic $\xi$-algebra, then $R$ is a subdirect sum of subdirectly irreducible algebras $R_{\alpha}$ where either (1) $R_{\alpha}$ is a division algebra or (2) there exists an ideal $P_{\alpha}$ of $R_{\alpha}$ contained in the center of $R_{\alpha}$ such that either $R_{\alpha}$ is commutative or $R_{\alpha} / P_{\alpha}$ is a field.

THeOREM 6A. If $R$ is an algebraic $\xi$-algebra over a perfect field, then $R$ is a subdirect sum of subdirectly irreducible algebras $R_{\alpha}$ which are either division algebras or commutative algebras.

We reproduce an example due to McLaughlin and Rosenberg [8, pp. 207-208] to show that the restriction that $\Phi$ is perfect is necessary in Theorem 6. At the same time, of course, we are furnished with a nontrivial illustration of Theorem 3 and Theorem 5 .

Let $\Phi$ be the two element field and $H=\Phi(x, y)$ the field of rational functions in two indeterminates $x$ and $y$ over $\Phi$. $J$ is the algebra $H+H a$, where multiplication is defined by:

$$
\left(g_{1}+h_{1} a\right)\left(g_{2}+h_{2} a\right)=\left(g_{1} g_{2}\right)+\left(h_{1} g_{2}+g_{1} h_{2}\right) a \text { for } g_{1}, g_{2}, h_{1}, h_{2} \in H .
$$

Finally $R$ is the algebra $J+J u+J v+J w$, with the multiplication table:

\begin{tabular}{|c|c|c|c|}
\hline & $u$ & $v$ & $w$ \\
\hline$u$ & $x$ & $w$ & $x v$ \\
\hline$v$ & $a+w$ & $y$ & $y u+a v$ \\
\hline$w$ & $a u+x v$ & $y u$ & $x y+a w$ \\
\hline
\end{tabular}

It is straightforward to check that $R$ is actually an associative algebra (of finite dimension) over $H$. It can be verified that $R$ is subdirectly irreducible with (using our usual notation) $(0) \neq S=a R=I=N$ 
$=A(S) \subset Z . R / A(S)$ is a field and $R$ is a $\xi$-algebra. We point out, finally, that $R$ is not commutative since, e.g., $u v-v u=-a \neq 0$.

\section{BIBLIOGRAPHY}

1. G. Birkhoff, Subdirect unions in universal algebra, Bull. Amer. Math. Soc. vol. 50 (1944) pp. 764-768.

2. N. Jacobson, The radical and semi-simplicity for arbitrary rings, Amer. J. Math. vol. 67 (1945) pp. 300-320.

3. - Structure theory for algebraic algebras of bounded degree, Ann. of Math. vol. 46 (1945) pp. 695-707.

4. I. N. Herstein, A generalization of a theory of Jacobson, Amer. J. Math. vol. 73 (1951) pp. 756-762.

5. - A generalization of a theorem of Jacobson. II, Bull. Amer. Math. Soc. Abstract 58-3-281.

6. - A generalization of a theorem of Jacobson. III, Amer. J. Math. vol. 55 (1953) pp. 105-111.

7. - The structure of a certain class of rings, Amer. J. Math. vol. 75 (1953) pp. 864-871.

8. J. E. McLaughlin and A. Rosenberg, Zero divisors and commutativity of rings, Proc. Amer. Math. Soc. vol. 4 (1953) pp. 203-211.

9. Y. Utumi, On $\xi$-rings, Proc. Japan Acad. vol. 33 (1957) pp. 63-66.

University of Pennsylvania and

YALE UNIVERSITY 